\title{
Archéopages
}

Archéopages Archéologie et société

$45 \mid 2017$

De la terre au pot

\section{Rien que des ratés !}

La production d'une faïencerie lilloise du XVIII ${ }^{\mathrm{e}}$ siècle à partir d'un dépotoir

Nothing but misfires! The production of Lille pottery of the eighteenth century found in a midden

¡Nada más que desechos! La producción de una fábrica de cerámica en Lille en el siglo XVII, a partir de un vertedero

\section{Vaiana Vincent et Ludovic Debs}

\section{(2) OpenEdition}

Journals

Édition électronique

URL : https://journals.openedition.org/archeopages/3393

DOI : $10.4000 /$ archeopages.3393

ISSN : 2269-9872

Éditeur

INRAP - Institut national de recherches archéologiques préventives

Édition imprimée

Date de publication : 1 mars 2018

Pagination : 110-117

ISSN : $1622-8545$

Référence électronique

Vaiana Vincent et Ludovic Debs, «Rien que des ratés! », Archéopages [En ligne], 45 | 2017, mis en ligne le 01 janvier 2020, consulté le 02 juin 2021. URL : http://journals.openedition.org/archeopages/3393 ; DOI : https://doi.org/10.4000/archeopages.3393 


\section{Rien que des ratés! \\ La production d'une faïencerie lilloise du XvIII siècle à partir d'un dépotoir}

Vaiana Vincent Inrap, UMR 6273, «Craham »

Ludovic Debs Irrap
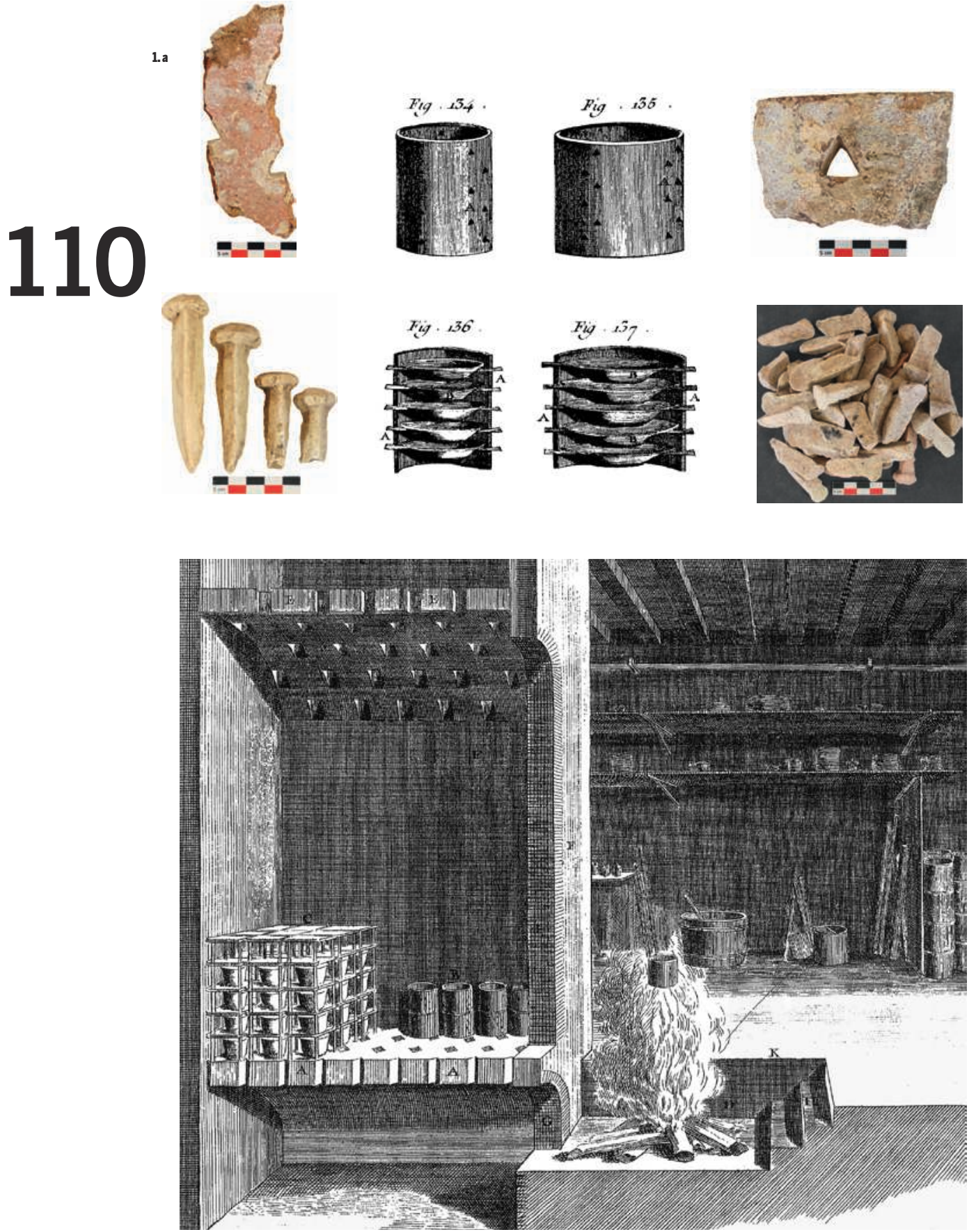

1.6
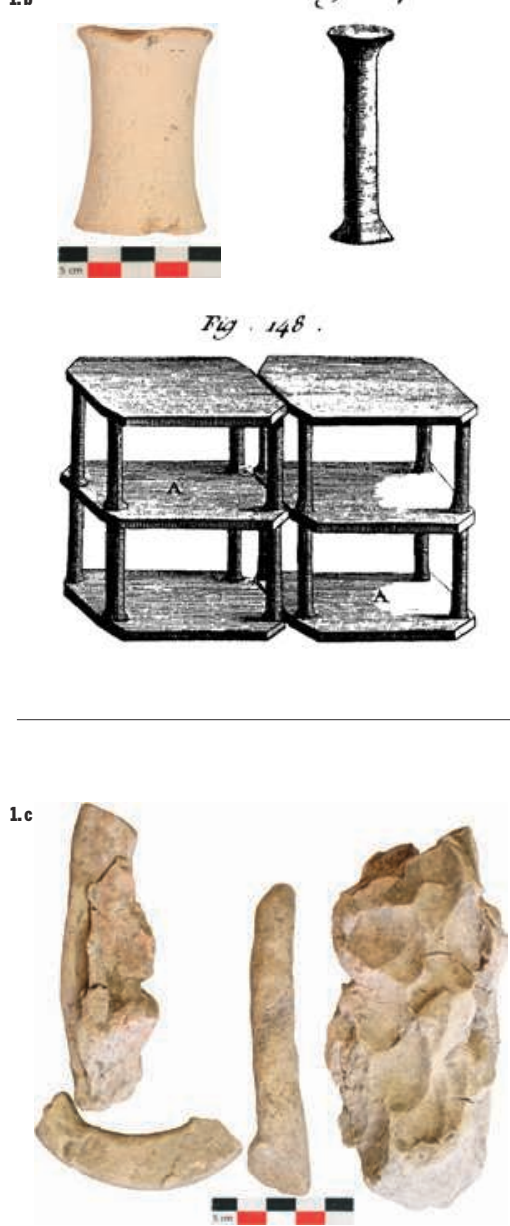
1. L'encyclopédie de Diderot et d'Alembert, sur l'u Art de la céramique ॥ (Diderot, AlemberT, 2002, article « Fayancerie ॥, PI.VIII et IX) est un excellent support de comparaisons pour l'art faïencier ou du moins pour comprendre les techniques utilisées dans l'atelier. Les systèmes de l'encastage (a) et de l'échappade (b) sont ainsi bien illustrés. La fouille a également mis au jour des boudins d'argile visant à colmater le couvercle de la cazette (c); le pâton d'argile sur la droite avec les nombreuses empreintes de doigt est destiné à caler les échafaudages dans le four.
Les faïenceries lilloises, de même que leur production, sont encore à ce jour très peu connues. Le rayonnement des productions de Delft, aux Pays-Bas, et celui de la manufacture de Rouen sont tels que, d'un point de vue historiographique, les recherches lilloises peinent à trouver leur place (Jacob, 1979 ; De Plas, 1977). Le second problème, découlant du premier, est la difficulté d'identification des faïences lilloises. En effet, seuls quelques objets peuvent être clairement rattachés à ces dernières. Les différents catalogues d'exposition consultés font part d'une attribution possiblement erronée d'objets assimilés, peut-être trop hâtivement, aux deux faïenceries susnommées. Les productions lilloises reprennent, en effet, de nombreux répertoires décoratifs de ces deux centres, ce qui rend très délicat leur identification. Cette méconnaissance se perçoit aussi à travers le biais muséal. À titre d'exemple, les pièces conservées au musée des Beaux-Arts de Lille ou au musée de la Manufacture nationale de Sèvres sont généralement celles présentant un décor élaboré. Elles constituent des objets exceptionnels qui ont traversé les siècles, suscitant l'intérêt des collectionneurs. Elles ne sont en aucun cas représentatives de l'essentiel de la production. Cette dernière est davantage composée d'objets du quotidien, utilitaires, destinés à la bourgeoisie, comme aux milieux plus modestes (Rosen, 1995, p. 129). Un catalogue d'exposition publié en 1994 sur les faïences du Nord indique clairement que la seule façon d'en savoir plus sur le sujet est de faire des fouilles archéologiques (Bonifas, 1994, p. 5).

\section{Un « glacis » de faïence}

Un diagnostic ${ }^{\mathbf{1}}$ a apporté des éléments nouveaux sur les faïences lilloises. L'opération archéologique portait sur une partie du Champ de Mars, longeant le côté est de la citadelle de Lille (Debs, 2011) [encadré 1]. Au-delà des découvertes liées au système défensif de la ville (mur de communication, " grand carré », demi-lune, lunette, contregarde et magasin à poudre), de nombreux déchets d'un atelier de faïencier ont été découverts, en position secondaire. Ceux-ci constituaient une épaisse couche de remblais, associés à de nombreux matériaux de démolition, qui venaient former le glacis de l'ouvrage fortifié. La mise en œuvre de ce glacis dispose d'un excellent terminus post-quem car il fait partie des modifications qui ont lieu après le siège de la ville de Lille en 1708, pendant la guerre de Succession d'Espagne. Le mobilier céramique se présente sous forme de couches denses et homogènes de déchets de fabrication et de rejets de production, visibles en coupe sur plusieurs mètres de long et jusqu'à $50 \mathrm{~cm}$ d'épaisseur. Étant donné la quantité retrouvée, l'ensemble a seulement été échantillonné. Seules les plus grosses pièces, les lèvres et les éléments présentant un décor ont été ramassés. La grande majorité du dépôt est constituée de biscuits ${ }^{2}$, et de plus rares tessons recouverts d'émail stannifère ${ }^{\mathbf{3}}$.
La présence de carreaux est aussi à noter. Ratés, éléments de cuisson, essais de peinture sont autant d'indices qui nous permettent de comprendre la chaîne opératoire de cette production de faïence.

\section{Un mode opératoire complexe}

La faïencerie utilise des techniques de fabrication qui lui sont propres. Les artefacts du dépôt permettent de comprendre certaines étapes de la chaîne opératoire de celle-ci. Les biscuits retrouvés relèvent de deux techniques de façonnage. La majorité d'entre eux sont manifestement tournés. Les assiettes, par exemple, ont une forme globalement identique mais présentent des mises en œuvre variables (inclinaison du marli, épaisseur de la pâte, courbure de la lèvre plus ou moins accentuée...) et surtout des traces de tournage visibles. Certains éléments sont obtenus avec la technique du moulage. C'est le cas pour une lèvre de « fontaine à la romaine », qui dispose d'une panse godronnée ou d'un couvercle, à décor de médaillons en léger relief. Une fois formées, les pièces subissent une première cuisson avant d'être recouvertes de leur émail et d'un éventuel décor.

Même si ce dépôt secondaire ne nous permet pas d'appréhender la topographie de l'atelier ou les types de fours utilisés, certains éléments nous renseignent sur le mode de cuisson. La fouille a ainsi mis au jour des fragments de cazettes, cylindres en terre cuite dans laquelle les pièces façonnées étaient disposées les unes sur les autres, afin d'être protégées « des flammes directes, des fumées et des cendres » (Rosen, 1995, p. 50), ainsi que de très nombreuses pernettes, fins boudins d'argile de section triangulaire qui, fichés dans les trous de la cazette prévus à cet effet, permettaient de séparer les pièces les unes des autres pour éviter leur fusion lors de la cuisson. La méthode de l'encastage, documentée dans l'Encyclopédie [ill. 1a], était donc utilisée pour enfourner certaines pièces. Les cazettes étaient généralement fermées d'un couvercle en terre cuite et colmatées à l'aide d'un boudin d'argile. Un grand nombre de ces boudins ont aussi été retrouvés dans cet ensemble. Une autre méthode d'enfournement a été mise en évidence. Il s'agit de l'échappade. Cette technique, réservée aux objets qui ne pouvaient être superposés, consistait à encastrer les pièces entre deux grandes tuiles séparées par de petites pilettes en terre cuite en forme de bobine [ill. 1b], nommées piliers d'échappade. Un seul pilier a été découvert lors de ce diagnostic. D'autres éléments plus anecdotiques peuvent aussi être cités : un pâton d'argile destiné à caler les échafaudages à l'intérieur du four ou des colombins d'argile pour séparer les carreaux les uns des autres. L'ensemble de ces indices prouve l'utilisation de techniques de production conformes à « l'idéal » présenté dans l'Encyclopédie.

Les biscuits ramassés lors du diagnostic portent différents tests de décor effectués par les peintres.
1. Réalisé en 2011

2. Poteries brutes non

recouvertes d'émail et

supérieure à $980^{\circ} \mathrm{C}$.

3. Mélange d'oxyde

de plomb et d'oxyd

d'étain qui fait toute

la particularité

de la faïence. 


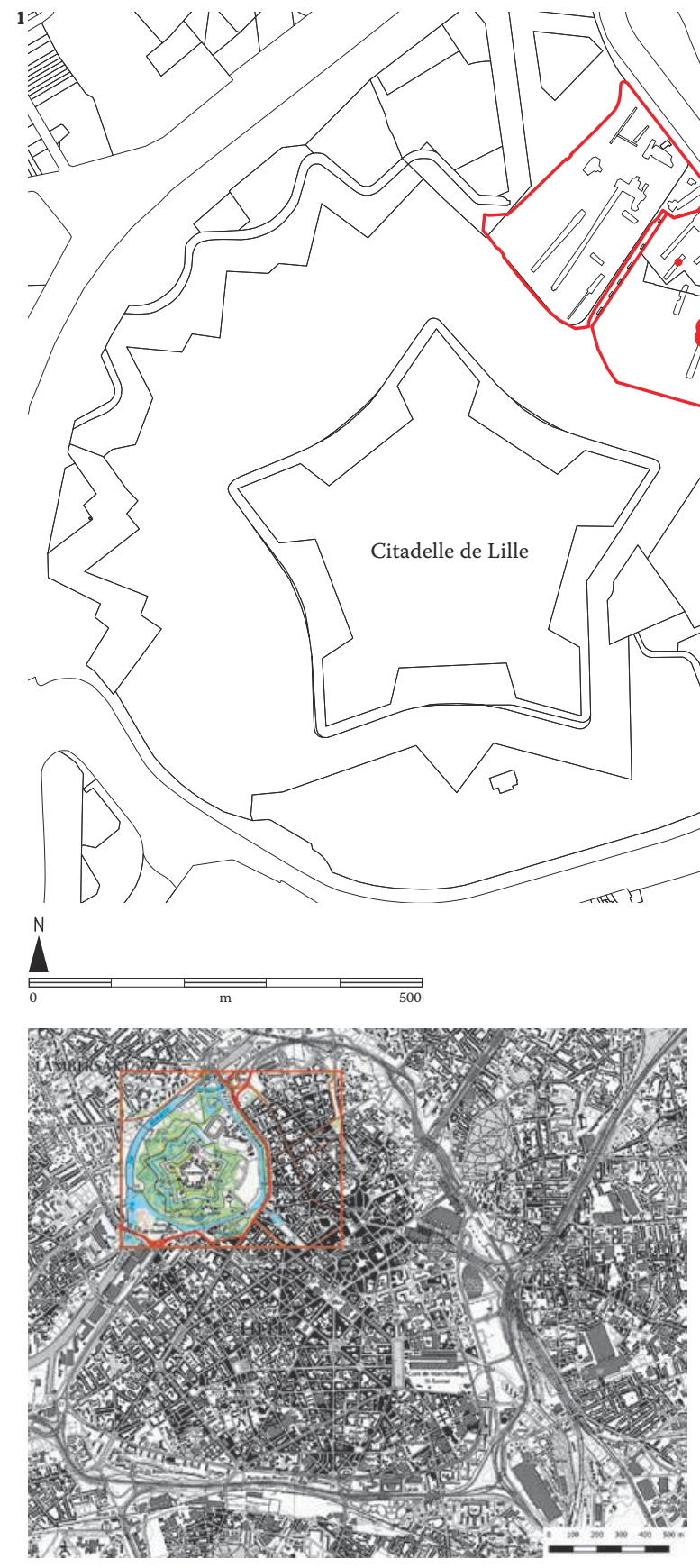

\section{Les faïenceries lilloises}

L'histoire des ateliers de faïences du Nord bénéficie dans la seconde moitié du $\mathrm{xIx}^{\mathrm{e}}$ siècle de l'intérêt d'amateurs éclairés (Bonifas, 1985, p. 11 ). Jules Houdoy écrit ainsi une Histoire de la céramique lilloise à partir d'un important dépouillement archivistique (Houdoy, 1869). La première faïencerie s'installe à Lille en 1696. Après la paix d'Aix-la-Chapelle en 1668 , le rattachement de Lille à la France permet un véritable renouveau industriel dans la région. En effet, il engendre la rupture des liens commerciaux traditionnels avec les Pays-Bas, ce qui occasionne un besoin en manufacture locale et notamment en faïencerie (Palais des Beaux-Arts de Lille, 1990, p. 11 1). C'est ainsi que Jacques Febvrier, natif de Tournai, et Jean Bossut, de Gand, deviennent les premiers "fabricateurs de faïence " à Lille (Houdoy, 1869, p. 27). Ils s'implantent dans une maison située rue Princesse, dans le Vieux-
Lille, à proximité du béguinage. Très rapidement, les deux associés se séparent et Febvrier garde la jouissance de la faïencerie. Quelques années plus tard, en 1711, en pleine occupation hollandaise, une seconde manufacture est créée sous l'égide de Barthélémy Dorez. Ce dernier est le seul à produire, en plus de la faïence, de la porcelaine tendre, très à la mode avec les importations chinoises et japonaises. Il fait une requête pour obtenir les locaux d'un ancien cabaret établi " quai du rivage du Haut ", mais obtient finalement une ancienne fabrique de sucre située « quai de la Haute Deûle ", actuel quai du Wault. Enfin, le dernier à s'installer est Jean-Baptiste Wamps en 1740. Spécialisé dans la fabrication des carreaux «à la manière de Hollande » (Houdoy, 1869, p. 92), il prend place rue de Metz. Si l'on considère l'implantation de ces trois ateliers [ill. 1], ainsi que la densité du matériel trouvé au sein des tranchées de diagnostic, il est permis de privilégier l'hypothèse que le dépotoir fouillé corresponde aux fabriques de Febvrier ou de Wamps. De plus, la nature même du dépôt qui n'est composé que de faïences ou de carreaux émaillés écarte obligatoirement la manufacture Dorez et sa porcelaine tendre. Une autre opération de diagnostic, qui s'est déroulée en 2012 au sud de la première (dirigée par L. Debs), a également mis au jour des déchets de faïencerie dont l'étude n'a encore pu être réalisée. Ce dépôt, plus proche de la manufacture de B. Dorez, sera un excellent comparatif pour l'ensemble présenté ici.

L'attribution du dépotoir à l'atelier de J.-B. Wamps qui, lui, fabrique uniquement des carreaux peut aussi être écartée. Il est alors possible de considérer que le dépotoir appartient probablement à la première fabrique, celle de J. Febvrier, fabricant à la fois de faïences et de carreaux, dont l'atelier est situé à $600 \mathrm{~m}$ de la citadelle.
1.Plan parcellaire de Lille, avec en rouge, l'emprise de l'opération dans laquelle est indiquée par un point rouge la densité plus ou moins importante des artefacts liés au rejet de la faïencerie. En vert, les implantations supposées des trois manufactures de Lille, avec leur nom et date d'installation. 

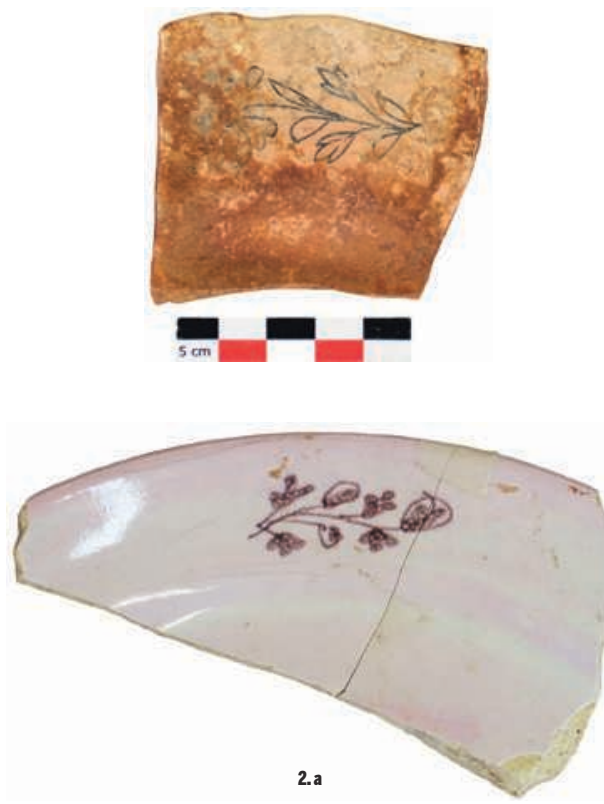

2. a

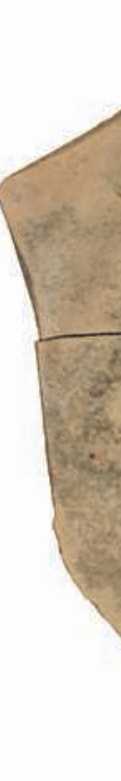

2.b

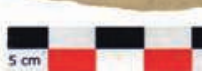

En effet, ces derniers réalisaient la plupart de leurs décors à main levée. Ils devaient donc s'exercer de nombreuses fois afin « d'acquérir l'automatisme du geste » (Rosen, 1995, p. 41). Un dessin de fines fleurs sur un biscuit se retrouve ainsi sur un produit fini. Ce dernier, peint à l'oxyde de manganèse, prend des teintes violacées [ill. 2a]. Sur un autre biscuit, est peinte une succession de chiffres qui peut s'apparenter à des chiffres de comptabilité, sorte d'ostracas modernes [ill. 2c]. La répétition du motif de la fleur de lys plus ou moins stylisée semble être utilisée comme une marque de la production [ill. 2b]. Il s'agit en fait du blason de Lille, « de gueules à la fleur de lys florencée d'argent », appelée également «fleur de Lille ». L'apposition de marques au revers des pièces est d'un usage courant dans les grandes fabriques (Lahaussois, 1998, p. 99). Elles indiquent les initiales du directeur, du propriétaire ou les sigles des manufactures.

\section{La nature de la production}

Une production céramique se définit en premier lieu par sa pâte. Étant donné que les différentes étapes de cuisson sont représentées (biscuits, objets faïencés), différentes teintes peuvent être observées, en fonction du degré de cuisson, allant du rosé clair au jaune puis crème [ill. 3]. Il s'agit d'une pâte avec une matrice assez fine, quasiment en début de vitrification. Quelques grains de quartz de fin module sont à noter, de même que d'autres plus rares et épars, de module moyen. Des petits nodules noirs d'origine minérale peuvent également être observés ponctuellement, ainsi que des oxydes de fer d'assez grosse dimension. La terre semble tirée du village de Bruyelles au sud de Tournai, d'après le dépouillement des archives effectué par Jules Houdoy au XIX ${ }^{\mathrm{e}}$ siècle. Ce village paraît approvisionner aussi les faïenceries dites de « Hollande », ce qui complique encore une fois l'identification des objets de diverses provenances, réalisés à partir d'une même matière première. On apprend toutefois dans l'inventaire de 1733 que l'argile provient également de « Grugeons » [encadré 2]. Gruson est un village situé à mi-chemin entre Lille et Tournai. L'approvisionnement en argile se fait donc à partir de plusieurs carrières, mais toujours à une échelle locale. Une fois encore, seuls les écrits nous permettent de comprendre l'économie de cet atelier. Il est répété à plusieurs reprises que l'étain provient d'Angleterre ; il serait d'une qualité inégalable et d'une grande blancheur.

Sans entrer dans une étude typologique, il est intéressant de comparer la proportion de formes identifiées lors du diagnostic et celles inventoriées lors de la liquidation de la faïencerie en 1698, après la séparation des deux premiers associés, Febvrier et Bossut (Houdoy, 1869, p. 34) [ill. 4]. Trois points peuvent être soulignés. Le premier est l'adéquation entre les données archéologiques et historiques sur l'importance des assiettes dans la production. Elles représentent en effet 30,4\% du corpus échantillonné et $28,2 \%$ des pièces de l'inventaire. La substantielle différence du pourcentage de plats peut s'expliquer par un problème de terminologie, les plats et les assiettes ayant une morphologie assez identique. Ont été considérés comme des 
La fabrique Febvrier-Boussemart

À la mort de J. Febvrier en 1729, sa veuve et son gendre François-Joseph Boussemart reprennent l'entreprise, d'où la dénomination de cette fabrique. Même si peu d'objets de cette époque sont connus, il semble que déjà l'entreprise était en pleine expansion et jouissait d'un certain succès. Les deux créateurs originels de la fabrique sont natifs des Pays-Bas espagnols et de nombreuses mentions dans les archives indiquent que les ouvriers venaient de Hollande, Gand et Rouen, ce qui peut expliquer les difficultés d'attribution de ces faïences influencées par les plus grands centres de production de l'époque.

Un document daté de 1733 est intéressant à plus d'un titre. Il s'agit d'une requête de la veuve Febvrier et de Boussemart qui souhaitent, à côté de leur manufacture de faïence, établir une verrerie.
Pour justifier l'aspect florissant de leur fabrique, ils en font une description précise, en détaillant la totalité de son contenu. On apprend ainsi les infrastructures présentes (nombre de fours, moulins, besoin en chevaux), la quantification des pièces réalisées, du besoin en bois, en étain et en plomb, le nombre d'ouvriers présents et leur fonction, mais aussi la quantité et l'origine des produits (bleu des Indes, soude d'Alicante ou sable de Gand par exemple). Étant donné l'objet de la requête, il faut prendre un certain recul face aux chiffres annoncés. Ce document n'en demeure pas moins exceptionnel pour la connaissance de l'organisation de travail, du choix des matériaux et des différentes phases de fabrication de cette faïencerie.

État de la manufacture de faïence de la veuve Febvrier et du sieur Boussemart, son gendre, en 1733

Extrait de Houdoy, 1869, p. 52-54.

Trois fours pour cuire la faïence, deux construits à la façon de Hollande, \& le troisième, qui est double en grandeur, cuisant au moins par an 1,287,60o pièces de faïence, toutes pièces utiles et recherchées par le public, de manière que l'on peut dire qu'il n'y a point une seule manufacture dans toute l'Europe où l'on fasse une aussi grande quantité de faïence. Ce nombre sera vérifié, au besoin, par le détail que donne chaque ouvrier, des ouvrages par lui faits.

Lesquels trois fours consomment, tous les ans, 60,00o faisceaux de bois-blanc.

Un quatrième four qui sert à calciner le plomb \& l'étain dont la consommation est, savoir : pour l'étain, de six mille livres, \& quant au plomb, de douze mille.

Deux grands moulins dont l'entretien est de six chevaux, composés de douze lanternes $\&$ meules servant à broyer les couleurs, tant en blanc qu'en bleu \& autres, chaque meule broyant cent cinquante livres de matières.

Terre et sable

Quatre cents charretés de marle de Grugeons,

Quatre bateaux de terre rouge tirée près la ville d'Aire,

Un bateau de sable venant du Saz de Gand

Un demi-bateau de terre noire qui se tire proche d'Arras,

Douze charretées d'argile,

Deux tonnes de saffre, qui est bleu des Indes, Quatre tonnes de bleu d'ampoisse,

Mille livres de potasse,

Quatre mille livres de soude d'Alicante,

Quatre cents livres de l'étarge d'or,
Deux cents livres de Périgor

tirées du Périgor,

Cent livres de rouge,

Six cents livres de mine de plomb,

Cents livres d'antimoine,

Cinq mille livres de sel blanc,

Sept mille livres de plomb affiné,

Deux cents livres de limures d'épingles,

Et cinquante autre choses non spécifiées

ci-dessus.

Douze moulins dont douze tourneurs, tournant l'un parmi l'autre trois cents pièces d'ouvrage chaque jour,

Un carreleur qui travaille au moins soixante mille carreaux de faïence par an ;

Quinze peintres pour la peinture des ouvrages qui sont mis en couleur \& qui gagnent, l'un parmi l'autre, cinquante patars par jour chacun (le patar valait six centimes un quart);

Un plombeur \& un manouvrier, qui mettent le blanc sur tous les ouvrages;

Cinq enfourneurs pour ranger les ouvrages dans les fours, \& dont trois sont aussi cuiseurs,

Deux laveurs de terre;

Quatorze manœuvriers dont l'emploi est de régler les ouvrages $\&$ de les mettre en état d'être cuits ;

Un estampeur de plombure et conducteur de chevaux;

Un fendeur de bois pendant l'année,

Un empailleur,

Et un nombre de neuf apprentis,

Et enfin le nombre de cent mille pièces ou environ, tant en caisses pour cuire la faïence, que mottes, carreaux, trépieds, jambes de chien, planches et autre outils à l'usage de ladite manufacture, non ici spécifiés. 


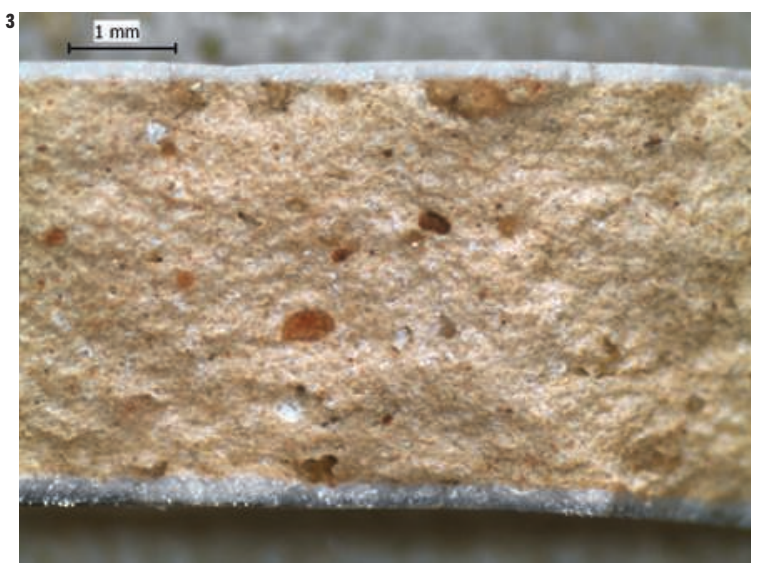

\begin{tabular}{|lllll|}
\hline formes & diag 2011 & \% diag 2011 & inv. 1698 & \% inv. 1698 \\
\hline plats & 187 & $37,9 \%$ & 137 & $7,7 \%$ \\
\hline assiettes & 150 & $30,4 \%$ & 500 & $28,2 \%$ \\
\hline bols & 93 & $18,8 \%$ & nd & - \\
\hline carreaux & 32 & $6,5 \%$ & nd & - \\
\hline pots à onguent & 13 & $2,6 \%$ & nd & - \\
\hline tasses & 1 & $0,2 \%$ & 327 & $18,4 \%$ \\
\hline tasses à café & 11 & $2,2 \%$ & 511 & $28,8 \%$ \\
\hline pichets & 7 & $1,4 \%$ & nd & - \\
\hline autres objets & nd & - & 301 & $16,9 \%$ \\
\hline total & 494 & $100,0 \%$ & 1776 & $100,0 \%$ \\
\hline
\end{tabular}
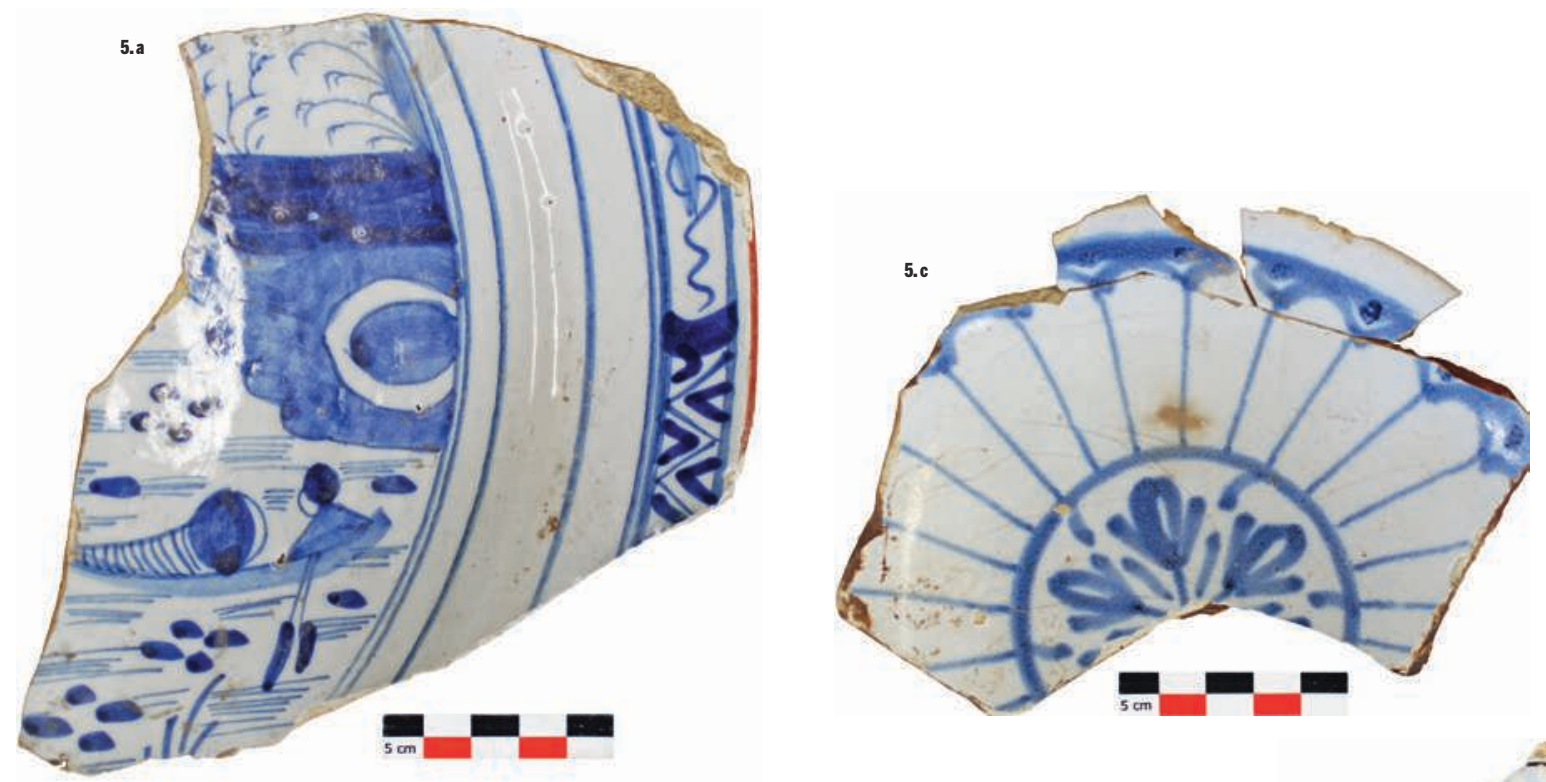

요
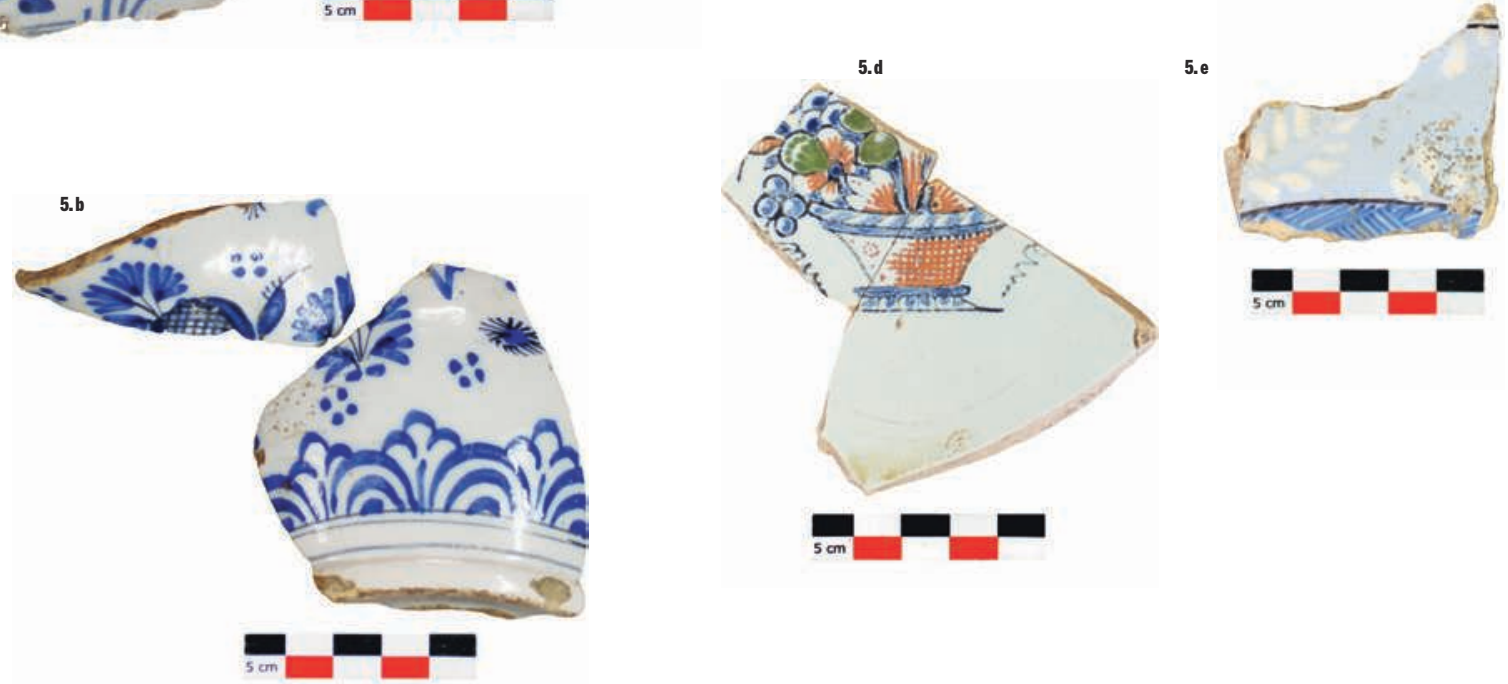

3. Cassure fraîche d'une

à la loupe binoculaire avec un grossissement $x 8$.
4. Comparaison du nombre de formes découvertes sur le diagnostic de 2011 avec I'inventaire de la liquidation de la faïencerie en 1698

(d'après Houdoy, 1869, p. 34). nd: information non disponible.
5. Les décors en camaïeu de bleu avec différents thèmes chinois (a), lambrequins (b) rayonnant (c), panier fleuri (d) ou bianco sopra bianco(e). 

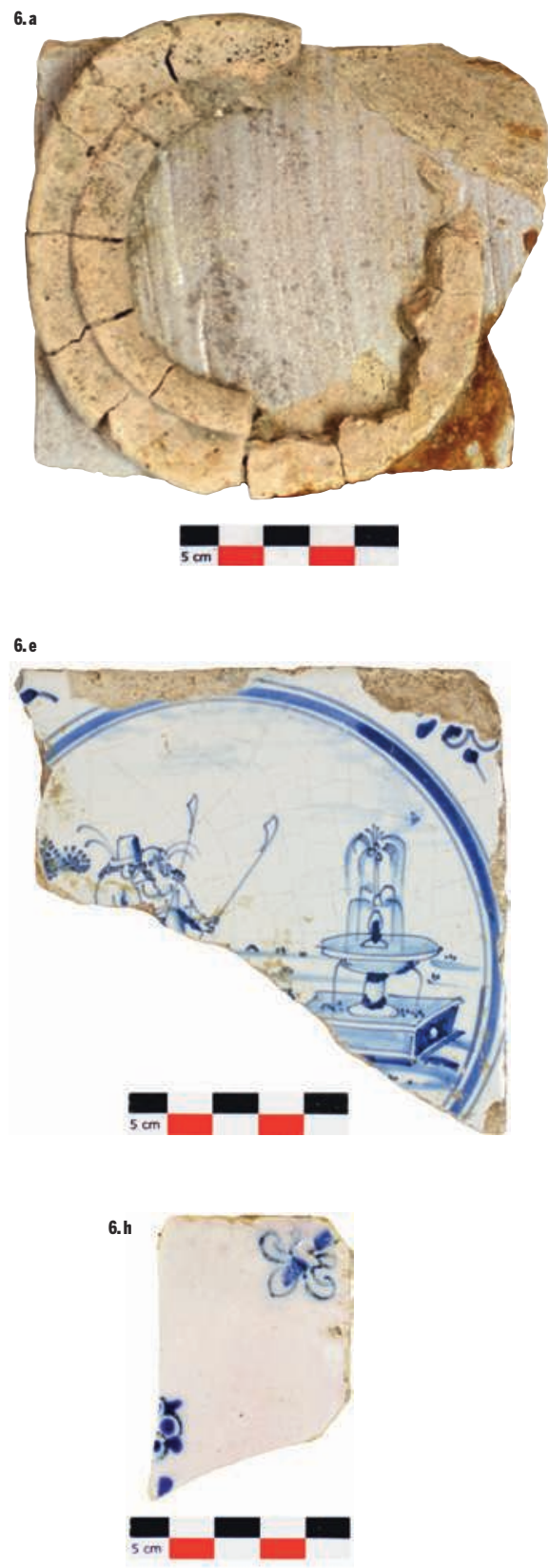

plats, dans l'étude archéologique, les objets de plus de $30 \mathrm{~cm}$ de diamètre, ce qui est tout à fait arbitraire. Le deuxième point est l'importance, dans l'inventaire de 1698, de la catégorie « autres objets » qui regroupe les «bénitiers, salières, petites urnes et moutardiers », totalement absents de la tessonnière. Enfin, le dernier point est la forte dissemblance de la proportion de tasses. Cela s'explique par la finesse de ces pièces, parfois presque comparable à de la coquille d'œuf. Leur caractère très cassant et le biais du ramassage archéologique expliquent leur sous-représentation ici.

Une production de faïence se caractérise enfin et surtout par son répertoire décoratif. Même si $84,5 \%$ du mobilier ramassé est constitué de biscuits et biscuits peints et 4,9\% de fragments de faïences blanches sans décor, $9,1 \%{ }^{\mathbf{4}}$ du matériel était émaillé et décoré, se rapprochant ainsi du
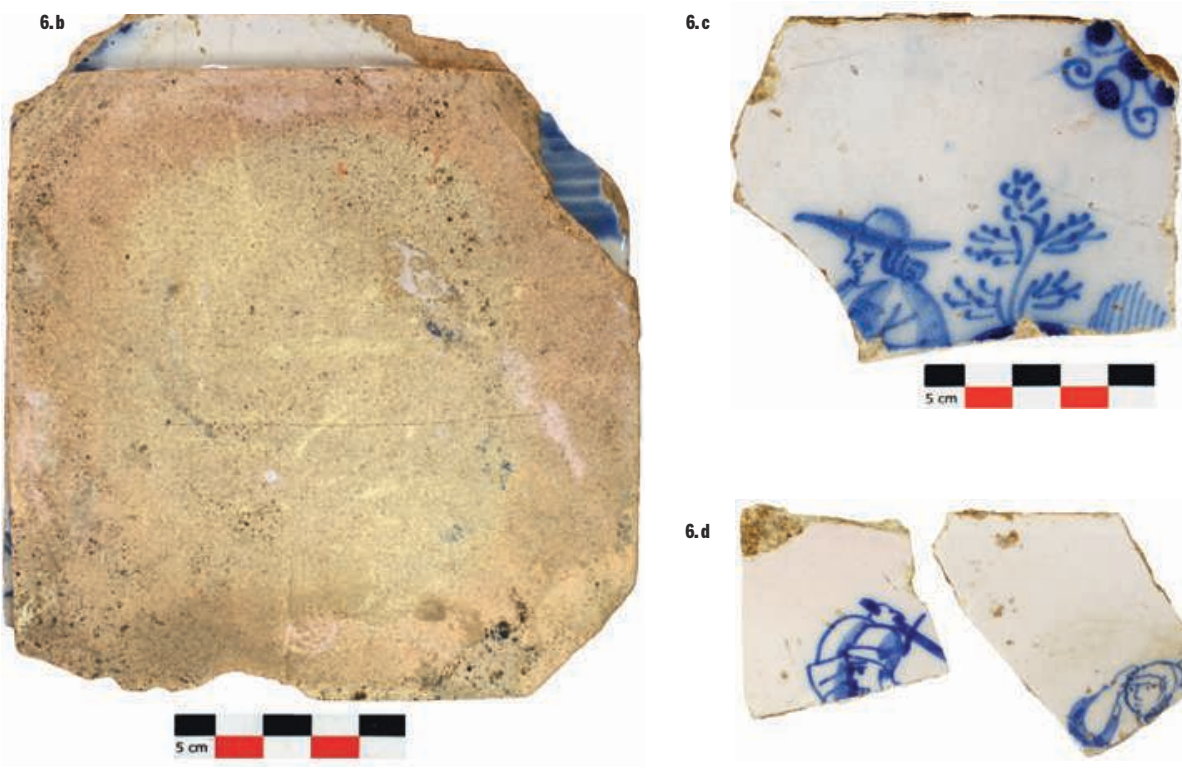

6.d
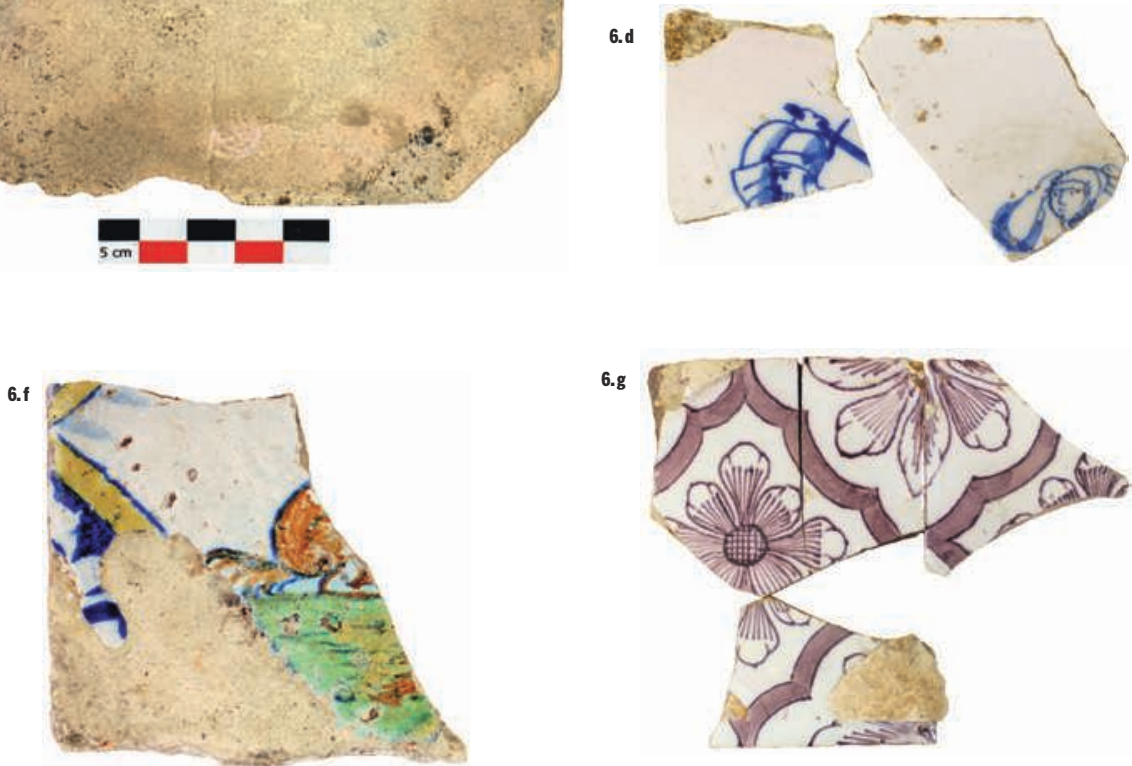

produit final voulu. Le décor est quasi

exclusivement composé d'un camaïeu de bleu, fortement inspiré des productions de Delft.

Les thèmes décoratifs sont variés [ill. 5]. On retrouve les décors dits « chinois » inspirés des productions delftoises qui voulaient copier les porcelaines exotiques, très en vogue dans la seconde moitié du XVII siècle (Faÿ-Hallé, Lahaussois, 2003, p. 158). L'autre type de décor répandu durant la première moitié du XVIII ${ }^{\mathrm{e}}$ siècle est le décor de broderies dit « au lambrequin », qui reprend des motifs des planches gravées des ornemanistes, artisans qui concevaient des ornements (Bonifas, 1994, p. 33). C'est de ce même mouvement que le décor rayonnant, bien représenté dans le corpus, tire aussi son inspiration. Le motif « à la corbeille fleurie », si caractéristique des productions rouennaises, est également utilisé sur un fond d'assiette. Un unique tesson présente un décor
4. Les $1,5 \%$ restant englobent de la céramique de consommation, non liée à la production (porcelaine chinoise, assiettes, tasses, bol et pot de chambre en céramique commune). 
particulier dit bianco sopra bianco qui consiste à peindre un décor blanc sur un fond légèrement teinté. Ce type d'ornementation a fait la réputation des faïences de Saint-Amand-les-Eaux à partir du milieu du XVIII ${ }^{\mathrm{e}}$ siècle. L'observation de la pâte semble indiquer qu'il s'agit pourtant bien d'une fabrication lilloise. Les décors en polychromie sont plus rares et toujours obtenus avec la technique du grand feu, c'est-à-dire que les oxydes colorants sont appliqués sur un émail stannifère «cru », dans lequel a été plongé le biscuit. Les couleurs vont du jaune au vert, en passant par le rouge ; on constate une certaine récurrence du violet obtenu à partir du manganèse.

\section{Une production à part : les carreaux de faïences} « à la façon de Hollande »

Les nombreux ratés retrouvés dans le dépotoir, carreaux collés face contre face ou boudins d'argile fusionnés sur le revers d'un carreau, indiquent clairement que cette fabrication de carreaux n'est pas anecdotique dans l'atelier. Ils représentent moins de $10 \%$ des objets collectés, mais cela peut être dû au caractère aléatoire de l'échantillonnage. La pâte utilisée est identique au reste de la production, de couleur jaune à crème. Là encore, la majorité des décors consistent en camaïeux de bleu [ill. 6]. Les décors sont principalement figuratifs : un personnage à chapeau joue de la flûte ; un autre, casqué et armé, représente un cavalier ; un couple ayant chacun une canne à la main se promène près d'une fontaine. Un unique exemple montre un décor polychrome avec ce qui semble être l'arrière-train d'un chien ou d'un loup. Le décor peut aussi être obtenu uniquement au manganèse, comme le montrent deux carreaux associés, avec un décor de fleur ancrée dans un losange lobé, rappelant des ornements orientaux. Les mouches dans les angles des carreaux représentent des fleurs de lys plus ou moins stylisées, peut-être encore une fois pour évoquer la ville de Lille.

L'étude de ce dépotoir de faïencerie montre que, même en observant un matériel homogène, dont l'origine de production semble clairement identifiée, il reste délicat d'en ressortir la spécificité. La volonté des potiers et des peintres était, dès l'origine, de faire des objets à la façon de « Hollande ou de Rouen ». Aussi, l'argile utilisée, les camaïeux de bleu, les motifs chinois sont autant d'éléments qui témoignent d'un but précis : imiter les productions de Delft. De même, la systématisation des lambrequins, les décors ponctués de rouge, ou le motif du panier fleuri sont produits dans le but de concurrencer les pièces rouennaises. Les imitations sont quasi parfaites, animées par une volonté de répondre à des phénomènes de mode précis, et aidées en cela par l'extrême mobilité des ouvriers (venant de Rouen, Nevers ou de Hollande). Le contexte de découverte, associé à la technique et au type de décor rencontré, permet de proposer pour ces faïences lilloises une datation située dans la première moitié $\mathrm{du} \mathrm{XVIII}^{\mathrm{e}}$ siècle et plus précisément dans le deuxième quart de ce siècle.

L'examen de ce type d'ensembles céramiques a donc un intérêt majeur. D'une part, il permet de renouveler les connaissances sur le sujet, souvent anciennes. D'autre part, il considère ces productions sous un autre angle que celui des musées, avec un vaisselier beaucoup plus large, correspondant davantage à la production commune qu'aux pièces d'exception. Le caractère aléatoire de l'échantillonnage, réalisé rapidement dans le contexte d'un diagnostic, rend les interprétations incertaines s'agissant d'un dépôt aussi important. Cette étude permet donc de montrer, si besoin était, l'intérêt que pourrait avoir un prélèvement exhaustif dans ce type de dépotoir ou, à défaut, l'intervention sur le terrain du céramologue à même de faire des choix de prélèvement en fonction des problématiques céramologiques.

\section{Références bibliographiques}

Bonifas J., 1985, Les Faïenciers de St-Amand au XVIII siècle, Saint-Amand-les-Eaux, Éditions du musée de Saint-Amand-Les-Eaux, 254 p.

Bonifas J., 1994, Faïences du Nord de la France, Paris, Réunion des Musées Nationaux, 280 p.

Debs L., 2011, Lille, Nord, Avenue du Petit Paradis, Lille, Esplanade de la citadelle et Grand carré, rapport d'opération, Inrap-SRA Nord-Picardie, $211 \mathrm{p}$.

Diderot D., AlemberT J. D', 2002, L'Encyclopédie : Arts de la céramique, Tours, Inter-Livres, 1 vol.

Fä̈-Hallé A., LAHAUSSOIS C., 2003, La faïence européenne au XVII siècle. Le triomphe de Delft, Paris, Réunion des Musées Nationaux, $287 \mathrm{p}$

Houdoy J., 1869, Histoire de la céramique lilloise, Paris, Auguste Aubry, 167 p

ЈАсов A., 1979, Faïences du Nord. 2 : Douai, Englefontaine, Ferrière, Hesdin, Lille, Paris, ABC décor, $90 \mathrm{p}$

Lahaussois C., 1998, Faïences de Delft, Paris, Réunion des Musées Nationaux, $311 \mathrm{p}$.

De Plas S., 1977, Les faïences de Rouen, du nord de la France et de la région parisienne, Paris, Ch. Massin, $83 \mathrm{p}$.

Palais des BeauX-Arts de Lille, 1990, L'Europe de la Fä̈ence : XVII ${ }^{e}$ et XVIII ${ }^{e}$ siècles dans les Collections du musée des Beaux-Arts de Lille, exposition, Lille, 22 octobre 1990-28 janvier 1991, Lille, Association des conservateurs de la région Nord-Pas-de-Calais, 254 p.

ROSEN J., 1995, La faïence en France du XIV $V^{e}$ au XIX $X^{e}$ siècle : histoire et technique, Paris, Éditions Errance, 215 p. 